\title{
Human herpesvirus type 6 and 7-associated diseases: an update
}

\begin{abstract}
Human herpesvirus type 6 (HHV 6) and 7 (HHV 7), two different herpesviruses of herpes family, particularly HHV 7 can cause roseola and several other associated diseases, such as neurological diseases, Graves' disease, Hodgkin's disease, Kaposi's sarcoma, etc. The pathological association of HHV 7 with pityriasis rosea is still controversial. HHV 6 mostly infects CD4+ T cells. Genomes of both variants of HHV 6 and 7 are closely related. TP53 gene plays the major role in restricting the production of HHV 6B messengerribonucleic acid (mRNA). Nevertheless, the roles of both HHV 6 and 7 in various dermatological disorders and several other clinical manifestations are still to be defined. Further investigations are urgently needed.
\end{abstract}

Keywords: Herpesvirus, Type 6, Human, Associated, Diseases, Human Herpesvirus 6, “p53" gene, Pityriasis rosea, DNA, Central nervous system
Volume 5 Issue I - 2017

\author{
Attapon Cheepsattayakorn, ${ }^{1,2,3}$ Ruangrong \\ Cheepsattayakorn ${ }^{4}$ \\ 'Editor-in-Chief, Journal of Human Virology and Retrovirology, \\ USA \\ ${ }^{2}$ Oth Zonal Tuberculosis and Chest Disease Center,Thailand \\ ${ }^{3} 5$ th Office of Disease Prevention and Control, Ratchaburi, \\ Department of Disease Control, Ministry of Public Health, \\ Thailand \\ ${ }^{4}$ Department of Pathology, Faculty of Medicine, Chiang Mai \\ University, Thailand
}

Correspondence: Attapon Cheepsattayakorn, I Oth Zonal
Tuberculosis and Chest Disease Center, I 43 Sridornchai Road
Changklan Muang Chiang Mai 50I00 Thailand,,Tel 6653 I40767,
6653 276364, Fax 6653 I40773, 6653 273590,
Email attapon1958@gmail.com, attaponche@yahoo.com

Received: January 29, 2017| Published: February 07, 2017

\begin{abstract}
Abbreviations: CI, Confidence Interval; CNS, Central Nervous System; CYP1A1, Cytochrome P450, Family 1, Subfamily A, Polypeptide 1; DNA, Deoxyribonucleic Acid; FPRP, False-Positive Report Probability; GD, Graves' Disease; GSTP1, Glutathione S-Transferase Pi 1; HHV, Human Herpesvirus; mRNA, Messenger Ribonucleic Acid; OR, Odd Ratio, p, Probability; PR, Pityriasis Rosea; TP53, Tumor Protein “ $p 53$ ” Gene; US, United States
\end{abstract}

\section{Introduction}

There are 8 different viruses of herpes family that affect human beings. ${ }^{1}$ Human herpesvirus type 6 and 7 (HHV 6 and 7) can cause roseola. ${ }^{1}$ Most of human beings are so common infected with these types at some ages. ${ }^{1}$ In 1986, HHV 6 was discovered by Gallo et al. ${ }^{2}$ CD4+ T cells are the major cell type infected by HHV 6. ${ }^{3,4}$ HHV 7 was discovered in 1990 by June et al. ${ }^{5}$ whereas Kempf reported that it was discovered in 1989 as a new member of the beta-herpesvirus subfamily. ${ }^{6}$ Braun et al. ${ }^{7}$ and Black \& Pellett $^{8}$ reported that the genomes of both variants of HHV 6 and HHV 7 were closely related, with $20 \%-75 \%$ nucleic acid homology depending on the genes being compared. HHV 6 and 7 are highly prevalent in the healthy population with a cumulative probability for seropositivity of almost $100 \%$ in young-aged group. ${ }^{9}$ Okuno et al. ${ }^{10}$ demonstrated that HHV 6 most commonly infects infants between the ages of 3 and 6 months as protection from maternal antibodies wanes. ${ }^{10}$ Braun et al. ${ }^{7}$ reported that by the age of 3 years, $90 \%$ of the United States (US) population has been infected with HHV $6^{7}$ whereas $90 \%$ of the US population reveals evidence of HHV 7 infection, by the age of 5 years. ${ }^{8}$ The most common peak age range of initial infection for HHV 7 is 18 months to 3 years of age that is slightly later than that for HHV $6 .{ }^{11}$ Both HHV 6 and 7 are transmitted among close contacts via the respiratory route. ${ }^{12}$ HHVs have been identified in the thyroid, which can be a reservoir of latent HHVs. ${ }^{13} \mathrm{HHVs}$ have been isolated from $72.22 \%$ of thyroid tissue blocks of patients with Hashimoto's thyroiditis and Graves' disease (GD). ${ }^{14}$ Role of HHV 6 as an etiological agent in the central nervous system (CNS) is unclear due to its presentation in the healthy individuals' brain tissue. ${ }^{15} \mathrm{HHV} 6$ has been associated with encephalitis, febrile convulsions, and exanthema subitum in infants and immunocompromised adults and may play a role in acute disseminated encephalomyelitis, Guillain-Barre' syndrome, and multiple sclerosis although HHV 6 is generally asymptomatic. ${ }^{15}$ HHV 7 has not been demonstrated to cause a specific disease, but it is associated with encephalitis and febrile convulsions. ${ }^{15}$ HHV 7, a causative agent in a variety of childhood maculopapular rashes, was identified in psoriasis and other inflammatory skin disorders. ${ }^{6}$ There are controversial data on pathogenetic association of HHV 7 with pityriasis rosea (PR), an exanthematic skin disorder as well as the association of HHV 7 with Hodgkin's disease, a lymphoma type or other primary cutaneous lymphoma. ${ }^{6}$ Some previous studies reported the association of HHV 7 with various epidemiologic forms of Kaposi's sarcoma via HHV 7-infected monocytic cells. ${ }^{6}$ In transplant recipients, HHV 7 has been identified as an emerging pathogen and may exacerbate graft rejection. ${ }^{6}$ HHV 7 could be an important pathogenic co-factor in inflammatory and neoplastic disorders die to its ability to induce cytokine production in infected cells. ${ }^{6}$

\section{Genetic polymorphisms}

Tumor protein " $p 53$ " (TP53) gene, major inducer of apoptosis or cell cycle arrest in response to genotoxic stress and other types of stress is much known about the mechanism by which viral proteins in transformed cells prevent the activities of $p 53 .{ }^{16}$ During HHV 6B infection, There is induction of significant accumulation of $p 53$ in both the cytoplasm and nucleus and determines the phosphorylation of $p 53$ at Ser392. ${ }^{17-19}$ TP53 inhibits HHV 6B replication and diminishes the viral cytopathic effects by restricting the production of HHV 6B mRNA. ${ }^{20}$ Anti-p53 antibodies were detected in about $4 \%$ of sera of the patients with suspicion of having autoimmune thyroid disease. ${ }^{21}$ These findings may due to participation of TP53 in the autoimmune process since DNA damage and apoptosis may be associated with autoimmune thyroid disease. ${ }^{21} \mathrm{~A}$ series of codifying genes of detoxification enzymes, including cytochrome 
P450, family 1 , subfamily A, polypeptide 1 (CYP1A1), in addition to $72 T P 53$ polymorphisms and glutathione S-transferase pi 1 (GSTP 1) are associated with an increased risk of GD. ${ }^{22}$ Variants of codon 72 (72TP53) and codon 47 of exon 4 of TP53 increase the risk for HHV 6 infection in renal transplant recipients. ${ }^{23}$ A previous study demonstrated that CYP1A1, GSTP1, and 72TP53 variants and the presence of HHV 7 were significant risk factors for GD (Power of calculation for $C Y P 1 A 1=63.8 \%, G S T P 1=100 \%$, and $72 T P 53=$ $48.5 \%){ }^{24}$ All these variant-disease associations remained noteworthy (false-positive report probability (FPRP) values were under 0.2 ). ${ }^{25}$ The power of calculation was $100 \%$ and the FPRP was 0.180 for the strong association between HHV 7 and GD. ${ }^{24} \mathrm{HHV} 7$ was much more prevalent, compared to HHV $6(12.85 \%)$ among patients with GD $(64.64 \%)$ than among controls $(14.08 \%) .{ }^{24}$ Increasing the risk of developing GD by more than three times among patients with HHV 7 infection $(\mathrm{OR}=3.133,95 \% \mathrm{CI}=1.959-5.011, p<0.0001) .{ }^{24} \mathrm{This}$ study also revealed that GSTP1 (OR $=14.726,95 \% \mathrm{CI}=2.689$ $80.659, p=0.0019$ for individuals under 25 years of age; and OR $=3.338,95 \% \mathrm{CI}=1.978-5.635, p<0.0001$ for individuals over 25 years of age) , CYP1A1 (OR $=1.800,95 \% \mathrm{CI}=1.109-2.923, \mathrm{p}=$ 0.0175 for individuals under 25 years of age; and OR $=1.812,95 \%$ $\mathrm{CI}=1.055-3.111, p=0.0312$ for individuals over 25 years of age), Arg/Pro 72p53 (OR $=0.506,95 \% \mathrm{CI}=0.337-0.760, \mathrm{p}=0.0010$ for individuals under 25 years of age), and Pro/Pro 72 TP53 (OR = $3.841,95 \% \mathrm{CI}=1.383-10.667, p=0.0098$ for individuals under 25 years of age) genotypes are the significant risk factors for GD among individuals over 25 years of age with $\mathrm{HHV} 7$ infection $(\mathrm{OR}=3.717,95$ $\% \mathrm{CI}=2.229-6.197, p<0.0001) .{ }^{24}$ There was no association between any of the investigated thyroid antibodies and HHV 7 infection. ${ }^{24}$ This study also reported that ophthalmopathic disease occurred more frequent in male patients $(50.81 \%)$, compared to female patients $(36.74 \%)\left(\chi^{2}, p=0.0474\right)$ and more than half $(50.50 \%)$ of these GD patients with ophthalmopathy were cigarette smokers. ${ }^{24}$

\section{Clinical aspects}

Primary infection with HHV 7 is most likely to be asymptomatic, but it can cause roseola-like illness ${ }^{26}$ as well as primary infection with HHV $6 .{ }^{27} \mathrm{HHV} 6$ can be associated with an unspecified febrile illness of childhood, ${ }^{28}$ whereas HHV 6B is particular subtype associated with roseola. ${ }^{29}$ Children with characteristically coin-sized erythematous macules or slightly elevated papules (roseola) on the neck and head typically present with high fever, usually without signs of upper respiratory tract infection. ${ }^{26}$ The onset of roseola characteristically appears on the third day of fever, often coinciding with resolution of the fever. ${ }^{26}$ Ganciclovir and foscarnet, either alone or in combination, can be used for the management of HHV 7 -associated CNS and neurological diseases. ${ }^{15}$ Prospective clinical trials are needed to evaluate the efficacy of foscarnet. ${ }^{15}$ Ganciclovir, foscarnet, and cidofovir have some activity against HHV 6 and 7 at high concentrations, but these drugs are of limited practical use due to the need to intravenously administered them or the potentially serious side-effects. ${ }^{12}$ Acyclovir and its derivatives have little antiherpesviral activity against HHV 6 and $7 . .^{30}$ Thus, patients with roseola and active HHV 6 replication should not be treated with antiherpesviral chemotherapy. ${ }^{12}$ Nevertheless, these antiherpesvirals should be considered in widespread systemic disease or end organ dysfunction. ${ }^{12}$ Currently, patients with PR, whether in cases with confirmed HHV 7 infection or refuted, should also not be treated with these drugs. ${ }^{12}$

\section{Conclusion}

More than 28 years after the discovery of HHV 6 and 7, there has been considerable progress in characterizing their genetic structures, virus-induced effects on infected host cells and in the development of diagnostic methods. However, the roles of HHV 6 and 7 in clinical features and various dermatological diseases, particularly the etiologies of both PR and serious drug-induced hypersensitivity reactions are still to be defined. Further studies are urgently needed.

\section{Acknowledgments}

None.

\section{Conflicts of interest}

None.

\section{References}

1. http://bodyandhealth.canada.com/channel/infection/herpes/herpesvirus-8-types

2. Salahuddin SZ, Ablashi DV, Markham PD, et al. Isolation of a new virus, HBLV, in patients with lymphoproliferative disorders. Science. 1986;234(4776):596-601.

3. Lusso P, Markham PD, Tschachler E, et al. In vitro cellular tropism of human B-lymphotropic virus (human herpesvirus 6). J Exp Med. 1988;167(5):1659-1670.

4. Takahashi K, Sonoda S, Higashi K, et al. Predominant CD4+ Tlymphocyte tropism of human herpesvirus 6-related virus. $J$ Virol. 1989;63(7):3161-3163.

5. Frenkel N, Schirmer EC, Wyatt LS, et al. Isolation of a new herpesvirus from human CD4+ T cells. Proc Natl Acad Sci USA. 1990;87(2):748-752.

6. Kempf W. Human herpesvirus 7 in dermatology: what role does it play? Am J Clin Dermatol. 2002;3(5):309-315.

7. Braun DK, Dominguez G, Pellett PE. Human herpesvirus 6. Clin Microbiol Rev. 1997;10(3):521-567.

8. Black JB, Pellett PE. Human herpesvirus 7. Rev Med Virol. 1999;9(4):245-262.

9. Krueger GR, Koch B, Leyssens N, et al. Comparison of seroprevalences of human herpesvirus -6 and -7 in healthy blood donors from nine countries. Vox Sang. 1998;75(3):193-197.

10. Okuno T, Takahashi K, Balachandra K, et al. Seroepidemiology of human herpesvirus 6 infection in normal children and adults. $J$ Clin Microbiol. 1989;27(4):651-653.

11. Wyatt LS, Rodriguez WJ, Balachandran N, et al. Human herpesvirus 7: antigenic properties and prevalence in children and adults. $J$ Virol. 1991;65(11):6260-6265.

12. Blauvelt A. Skin diseases associated with human herpesvirus 6,7 , and 8 infection. J Investig Dermatol Symp Proc. 2001;6(3):197-202.

13. Chen T, Hudnall SD. Anatomical mapping of human herpesvirus reservoirs of infection. Mod Pathol. 2006;19(5):726-737.

14. Thomas D, Liakos V, Michou V, et al. Detection of herpesvirus DNA in post-operative thyroid tissue specimens of patients with autoimmune thyroid disease. Exp Clin Endocrinol Diabetes. 2008;116(1):35-39.

15. Dewhurst S. Human herpesvirus type 6 and human herpesvirus type 7 infections of the central nervous system. Herpes. 2004;11(Suppl 2): $105 \mathrm{~A}-111 \mathrm{~A}$.

16. Harris SL, Levine AJ. The p53 pathway: positive and negative feedback loops. Oncogene. 2005;24(17):2899-2908.

17. Takemoto M, Mori Y, Ueda K, et al. Productive human herpesvirus 6 infection causes aberrant accumulation of $\mathrm{p} 53$ and prevents apoptosis. $J$ Gen Virol. 2004;85(Pt 4):869-879. 
18. Oster B, Bundgaard B, Höllsberg P. Human herpesvirus 6B induces cell cycle arrest concomitant with p53 phosphorylation and accumulation in T cells. J Virol. 2005;79(3):1961-1965.

19. Oster B, Bundgaard B, Hupp TR, et al. Human herpesvirus 6B induces phosphorylation of p53 in its regulatory domain by a CK2- and p38independent pathway. J Gen Virol. 2008;89(Pt 1):87-96.

20. Oster B, Kofod-Olsen E, Bundgaard B, et al. Restriction of human herpesvirus 6B replication by p53.J Gen Virol. 2008;89(Pt 5):1106-1113.

21. Fenton CL, Patel A, Tuttle RM, et al. Autoantibodies to p53 in sera of patients with autoimmune thyroid disease. Ann Clin Lab Sci. 2000;30(2):179-183.

22. Bufalo NE, Santos RB, Cury AN, et al. Genetic polymorphisms associated with cigarette smoking and the risk of Graves' disease. Clin Endocrinol (Oxf). 2008;68(6):982-987.

23. Leite JL, Manfrinatto JA, Mazzali M, et al. Polymorphisms at exon 4 of p53 and the susceptibility to herpesvirus types 6 and 1 infections in renal transplant recipients. Transpl Int. 2006;19(9):732-737.

24. Leite JL, Bufalo NE, Santos RB, et al. Herpesvirus type 7 infection may play an important role in individuals with a genetic profile of susceptibility to Graves' disease. Eur J Endocrinol. 2010;162(2):315-321.
25. Bufalo NE, Leite JL, Guilhen AC, et al. Smoking and susceptibility to thyroid cancer : an inverse association with CYP1A1 alleic variants. Endocr Relat Cancer. 2006;13(4):1185-1193.

26. Tanaka K, Kondo T, Torigoe S, et al. Human herpesvirus 7: another causal agent for roseola (exanthem subitum). J Pediatr. 1994;125(1):1-5.

27. Yamanishi K, Okuno T, Shiraki K, et al. Identification of human herpesvirus-6 as a causal agent for exanthem subitum. Lancet. 1988;1(8594):1065-1067.

28. Chiu SS, Cheung CY, Tse CY, et al. Early diagnosis of primary human herpesvirus 6 infection in childhood: serology, polymerase chain reaction, and virus load. J Infect Dis. 1998;178(5):1250-1256.

29. Schirmer EC, Wyatt LS, Yamanishi K, et al. Differentiation between two distinc classes of viruses now classified as human herpesvirus 6. Proc Nalt Acad Sci USA. 1991;88(13):5922-5926.

30. Takahashi K, Suzuki M, Iwata Y, et al. Selective activity of various nucleoside and nucleoside analogues against human herpesvirus 6 and 7. Antiviral Chemistry \& Chemotherapy. 1997;8(1):24-31. 